\title{
Numerical solution of free-boundary problems in fluid mechanics. Part 2. Buoyancy-driven motion of a gas bubble through a quiescent liquid
}

\author{
By G. RYSKIN $\dagger$ AND L. G. LEAL \\ Department of Chemical Engineering, California Institute of Technology, \\ Pasadena, California 91125
}

(Received 11 April 1983 and in revised form 27 April 1984)

In this paper numerical results are presented for the buoyancy-driven rise of a deformable bubble through an unbounded quiescent fluid. Complete solutions, including the bubble shape, are obtained for Reynolds numbers in the range $1 \leqslant R \leqslant 200$ and for Weber numbers up to 20 . For Reynolds numbers $R \leqslant 20$ the shape of the bubble changes from nearly spherical to oblate-ellipsoidal to spherical-cap depending on Weber number; at higher Reynolds numbers 'disk-like' and 'saucer-like' shapes appear at $W=O(10)$. The present results show clearly that flow separation may occur at a smooth free surface at intermediate Reynolds numbers; this fact suggests a qualitative explanation of the often-observed irregular (zigzag or helical) paths of rising bubbles.

\section{Introduction}

The buoyancy-driven motion of a deformed bubble through a quiescent fluid has been extensively studied by experimentalists (see references in $\S 3$ ), but no satisfactory theory exists except for the cases of very small deformation at either high (Moore 1959,1965 ) or low ('Taylor \& Acrivos 1964; Brignell 1973) Reynolds number. The well-known analysis of the 'spherical-cap' bubble due to Davies \& Taylor (1950) relates the speed of rise to the radius of curvature of the bubble at the front stagnation point (Collins 1966), but the overall spherical-cap shape is assumed a priori rather than being determined as part of a full solution. The recent boundary-integral calculations of Miksis, Vanden-Broeck \& Keller (1981) are limited to potential flow, and thus do not allow for the possibility of separation in the wake. Later the same authors (1982) attempted to include viscous effects, but separation was still ignored.

In addition to being an inherently interesting physical problem, the motion of a deformable bubble also represents a good example of the important class of free-boundary problems of fluid mechanics, from which one may anticipate obtaining a better understanding of both solution methods and the factors that control the boundary shape. The problem also affords the opportunity of investigating the rather poorly understood phenomenon of flow separation at a free surface. The practical importance of bubble motions, ubiquitous as they are in nature and technology, is self-evident.

This paper represents the second in a three-part series on the numerical solution of free-boundary problems at finite Reynolds number by a finite-difference scheme

$\dagger$ Present address: Department of Chemical Engineering, Northwestern University, Evanston, Illinois 60201 . 
that is based upon the use of boundary-fitted, orthogonal coordinates that are generated as a part of the overall solution. The numerical technique was described in Part 1 of this series (Ryskin \& Leal 1984). Here we consider the problem of a deformed bubble rising under the action of gravity for Reynolds numbers in the range $1 \leqslant R \leqslant 200$ and Weber numbers up to 20 .

\section{Problem statement}

The gas bubble is assumed to be of constant volume $\frac{4}{3} \pi a^{3}$, rising under gravity with velocity $U$ in a quiescent incompressible Newtonian fluid of viscosity $\mu$ and density $\rho$. The density and the viscosity of the gas inside the bubble are assumed to be negligible in comparison with those of the liquid, and the interface of the bubble is assumed to be characterized by a constant surface tension $\gamma$ (thus excluding surfactant effects from consideration). In Part 1 we have presented the governing differential equations and boundary conditions, all expressed in terms of the curvilinear, orthogonal, boundary-fitted coordinates $(\xi, \eta, \phi)$, whose utilization is the basis for the finite-difference method that we have developed for this class of problem. In the present application, the Weber number that appears in the normal stress balance (equation (9) of Part 1) is defined as $W \equiv 2 \rho U^{2} a / \gamma$, and the Reynolds number is $2 \rho U a / \mu$. We restrict our attention to steady axisymmetric bubble shapes - which are physically realizable in experimental studies up to $R=O(100-200)$ or even higher, depending upon the Weber number.

The numerical algorithm that was used to solve the problem was described in detail in Part 1, as well as various checks that were made to test the accuracy of the resulting solutions. The only detail that is specific to the present problem and was not discussed in Part 1 is the equation for pressure at the bubble surface that is needed in applying the normal-stress balance. This is obtained by integrating the $\eta$ component of the equation of motion in the form

$$
\boldsymbol{\nabla}\left(p+u^{2}\right)=\frac{2 a g}{U^{2}} i_{x}+2(u \wedge \omega)-\frac{4}{R} \operatorname{curl} \omega
$$

evaluated at the bubble surface. Here $i_{x}$ is the unit vector in the direction of gravity, and the characteristic pressure is $p_{\mathrm{c}}=\frac{1}{2} \rho U^{2}$. The hydrostatic (body-force) term can be expressed in terms of the drag coefficient using the steady-state balance between the buoyancy force and the hydrodynamic drag,

$$
\frac{2 a g}{U^{2}}=\frac{3}{4} C_{\mathbf{D}}
$$

where

$$
C_{\mathrm{D}} \equiv \frac{\mathrm{drag}}{\frac{1}{2} \rho U^{2} \pi a^{2}}
$$

Thus the integrated form of (1), incorporating (2), is

$$
p=\frac{3}{4} C_{\mathrm{D}} x-u_{\eta}^{2}-\frac{4}{R} \int \frac{f}{\sigma} \frac{\partial}{\partial \xi}(\sigma \omega) \mathrm{d} \eta+\text { const },
$$

in which ${ }_{4}^{3} C_{\mathbf{D}} x$ is the hydrostatic pressure contribution $p_{\mathrm{hs}}$, and the remainder is the dynamic-pressure term $p_{\mathrm{dyn}}$.

It may be noted that the expression (3) for the pressure contains a free constant of integration, which is a consequence of the incompressibility assumption. This constant is determined in the numerical computation from the constraint (of incompressibility) that the bubble volume be constant as described in Part 1. 
The drag coefficient, required in (3), is calculated from the equation

$$
C_{\mathrm{D}}=2 \int_{0}^{1}\left(-p_{\mathrm{dyn}}+\frac{8}{R} e_{\xi \xi}\right) \sigma \frac{\partial \sigma}{\partial \eta} \mathrm{d} \eta
$$

Before describing the results of our numerical computations, we briefly review the existing experimental observations in the next section.

\section{Summary of experimental observations}

The experiments of Haberman \& Morton (1953), Saffman (1956), Hartunian \& Sears (1957), Hnat \& Buckmaster (1976), Bhaga \& Weber (1981) and others provide a fairly detailed picture of the motion of gas bubbles through a quiescent viscous liquid. It is convenient to describe their results in terms of the dimensionless parameter $M \equiv g \mu^{4} / \rho \gamma^{3}$, which depends only on liquid properties and is related to our parameters according to

$$
M=\frac{3}{4} C_{\mathrm{D}} \frac{W^{3}}{R^{4}}
$$

The variation of $M$ in pure liquids is mainly due to variations in viscosity; $M$ varies from $O\left(10^{-13}\right)$ in liquid metals to $O\left(10^{5}\right)$ in viscous oils. Water has a value of $M=O\left(10^{-10}\right)$.

In high- $M$ liquids ( $M \geqslant 4 \times 10^{-3}$ according to Bhaga \& Weber 1981), the flow past a bubble is steady if $R \leqslant 110$ (for $R>110$ the wake becomes unsteady) and the shape depends mainly on $R$, changing from spherical for $R \leqslant 0.1$ to oblate ellipsoidal, to ellipsoidal cap, and finally to spherical cap (Hnat \& Buckmaster 1976; Bhaga \& Weber 1981).

In low- $M$ liquids the bubbles likewise change from spherical to oblate-ellipsoidal at low $R$ and have a spherical-cap shape at large $R$. However, the transition between these regimes does not pass through a sequence of steady shapes as in the high- $M$ case. First, when the bubbles are still only slightly deformed at $W=O(3)$ and $R \geqslant 200$, their path becomes zigzag or helical (Hartunian \& Sears 1957). At higher $R$ and $W$ the path becomes rectilinear again, but the bubble becomes very flattened in shape, and fluctuates violently (Saffman 1956). Finally, at $W \geqslant O(30)$, the bubble assumes a stable spherical-cap shape, but with a very sharp (often ragged) rim and an unsteady or turbulent wake (Wegener \& Parlange 1973).

Our numerical solution technique is restricted to steady and axisymmetric laminar flows. In effect, we attempt to obtain steady axisymmetric solutions for both lowand high- $M$ conditions without questioning their stability (and hence realizability) in the real, physical situation. Although a failure of the numerical procedure to converge may sometimes be indicative of a physical instability, this is by no means obvious and should not be automatically construed in that light.

\section{Results of the numerical computations}

We have computed solutions for $R$ in the range $1 \leqslant R \leqslant 200$ and $W$ from 0 up to 20 for $R \leqslant 20$ and up to 10 for $R \geqslant 50$. In addition, several solutions were obtained for $R=0.5$ for comparison with the low-Reynolds-number small-deformation theories of Taylor \& Acrivos (1964) and Brignell (1973). The upper limits on $W$ are a consequence of the very large values of curvature which occur at the rim of the bubble, in combination with the use of a $40 \times 40$ mesh for the present calculations. 


$\begin{array}{lcc} & \text { Present results } & \text { Taylor \& Acrivos (1964) } \\ R=0.5, W=0 & 33.6 & 33.7 \\ R=0.5, W=0.5 & 34.7 & 35.1 \\ R=0.5, W=1.0 & 35.3 & 36.4\end{array}$

TABLE 1. Comparison of the present results for the drag coefficient at low $R$ and $W$ with the asymptotic theory of Taylor \& Acrivos (1964)

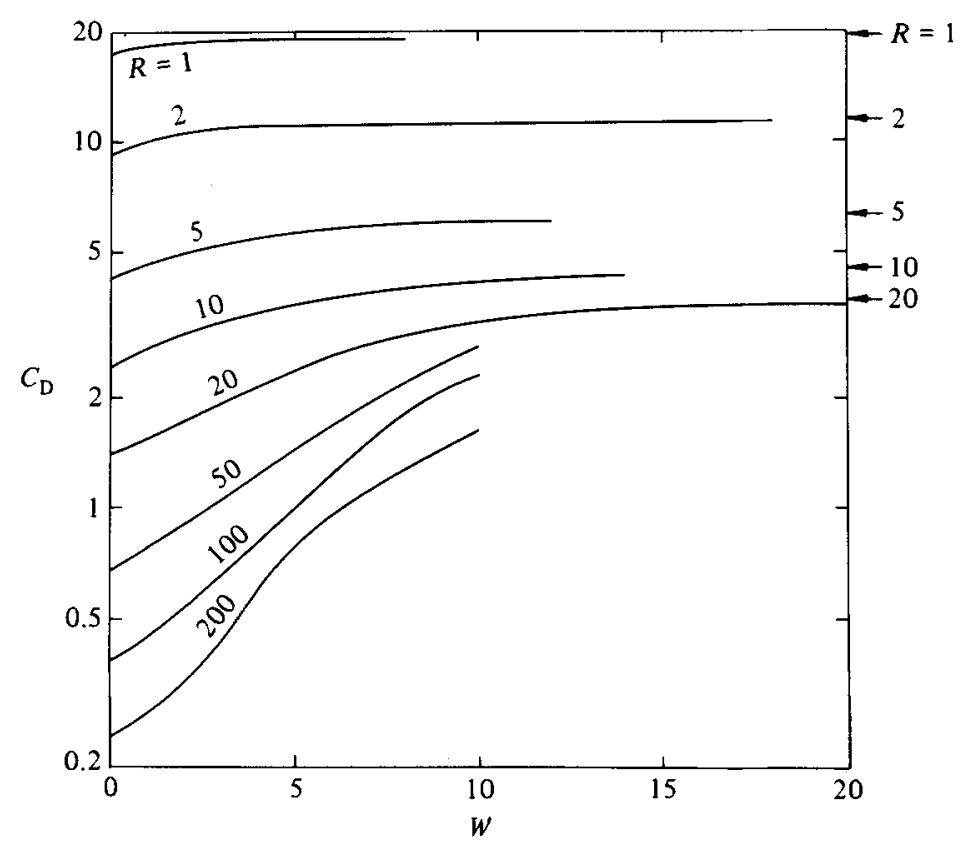

Figure 1. Drag coefficients for a steadily rising bubble as a function of the Weber number for various values of the Reynolds number. The arrows indicate the empirical correlation of Bhaga \& Weber (1981) for very large $W ; W=0$ corresponds to spherical bubbles.

\subsection{Drag coefficients}

Our results for the drag coefficient at $R=0.5$ are presented in table 1 , and of $C_{\mathrm{D}}$ for other values of $R$ and $W$ in figure 1 . A table with the actual numerical values of $C_{\mathrm{D}}$ at each $R$ and $W$ was also given in Part 1 , where the results were used to discuss the accuracy of our numerical procedures. The comparison in table 1 between the numerically calculated drag coefficients at $R=0.5$ and the values from Taylor \& Acrivos (1964) suggests that the asymptotic theory is quite accurate for $W \leqslant 0.5$, but somewhat less accurate for $W=1$. However, we shall discuss the limits of the asymptotic theory in more detail in $\$ 4.2$, where we consider the comparison between numerical and asymptotic bubble shapes.

The only prior numerical results for the drag on a bubble were for spherical bubbles by Brabston \& Keller (1975) and Rivkind \& Ryskin (1976). Drag coefficients for a spherical bubble were also obtained here as a byproduct of starting our calculations at each value of $R$ with a spherical shape (the values shown for $W=0$ in figure 1). These results are in essential agreement with the results of Brabston \& Keller (1975) 
and Rivkind \& Ryskin (1976). The latter authors also demonstrated good agreement with experimental data for cases where the actual bubble shapes are nearly spherical.

Bhaga \& Weber (1981) found experimentally that the drag coefficient (and shape) depended only on $R$ for $M \geqslant 4 \times 10^{-3}$ (i.e. for large- $M$ liquids in their language). Thus, referring to (5), it is evident that the drag coefficient and bubble shape should become independent of $W$ (for any $R$ ) as soon as $W$ is large enough to obtain $M \geqslant 4 \times 10^{-3}$. Using (5) and the numerical results for $C_{\mathrm{D}}$ in figure 1 (or table 1 of Part 1), we can, in fact, be more explicit as to which of the cases we have considered corresponds to ' low- $\boldsymbol{M}$ ' or ' high- $\boldsymbol{M}$ ' fluids respectively, based on the Bhaga \& Weber definition of large- $M$ liquids as being those with $M \geqslant 4 \times 10^{-3}$. In particular, all of our cases with $R \leqslant 10$, except $W \equiv 0$, have $M>4 \times 10^{-3}$, and thus represent high- $M$ results. All of our cases with $R \geqslant 50$, on the other hand, have $M<4 \times 10^{-3}$, and thus can be classified as low-M cases. Finally, for $R=20$ the transition between high $M$ and low $M$ occurs between $W=6$ and 8 . Going back now to Bhaga \& Weber's qualitative observation, it is thus evident that the drag coefficients for $R \leqslant 10$ should be approximately constant for all $W$ (except $W=0$ ), while, for $R=20, C_{\mathrm{D}}$ should become independent of $W$ for $W$ between 6 and 8 . Finally, for $R \geqslant 50$ we should not expect $C_{\mathrm{D}}$ to achieve an asymptote for any value of $W$ studied here. It is evident upon examination of figure 1 that our numerical results are in qualitative accord with Bhaga \& Weber's observations, though the cases $R=\mathbf{5}$ and 10 exhibit a weak dependence on $W$ over a wider range of $W$ than might have been anticipated on this basis. It may also be noted that Bhaga \& Weber (1981) gave an empirical formula for the drag coefficient for high $M$, namely

$$
C_{\mathrm{D}}=\left((2.67)^{0.9}+\left(\frac{16}{R}\right)^{0.9}\right)^{1 / 0.9}
$$

and the values of $C_{\mathrm{D}}$ calculated from this formula are shown on the right-hand side of figure 1 . For $R \leqslant 20$ and sufficiently large $W$ there is obviously good agreement between our results and the empirical large- $M$ formula of Bhaga \& Weber (1981).

Experimental data corresponding to $R \geqslant 50$ and $W \leqslant 10$ do not apparently exist. Such cases correspond to low- $M$ liquids with $O\left(10^{-9}\right)<M<O\left(3 \times 10^{-4}\right)$. In general, the experiments would be more difficult than those with high- $M$ liquids, because the small bubbles required to achieve $R$ in the range 50-200 would be highly susceptible to the influence of surface-active impurities. In addition, even if such experiments did exist, it is quite possible that the bubbles at these $R$ and $W$ would rise in zigzag or spiral path, thus rendering any comparison with the present solutions meaningless.

We have presented our results in this section in the form $C_{\mathrm{D}}=C_{\mathrm{D}}(R, W)$. It has been pointed out on numerous occasions that a more 'natural' representation of results would be to plot the dimensionless velocity $\bar{U} \equiv U /(\nu g)^{\frac{1}{3}}$ as a function of the dimensionless (equivalent) bubble radius $\bar{a} \equiv a /\left(\nu^{2} / g\right)^{\frac{1}{3}}$ for various fixed values of $M$. With this representation, the function $\bar{U}=\bar{U}(\bar{a} ; M)$ reduces to a universal curve, independent of $M$, for both the asymptotic limits of very small bubbles in the Hadamard-Rybczynski regime where $\bar{U}=\frac{1}{3} \vec{a}^{2}$ and very large spherical-cap bubbles in the Davies-Taylor regime where $\bar{U} \approx \bar{a}^{1}$. For the intermediate regime there is a monotonic increase in $\bar{U}$ with $\bar{a}$ in high- $M$ liquids. For the low-M case, however, $\bar{U}$ first increases with $\bar{a}$, but then decreases over some range of $\bar{a}$ before joining the spherical-cap asymptote (Haberman \& Morton 1953; Bhaga \& Weber 1981). Unfortunately, in the present work we have not solved a sufficiently large number of cases to obtain meaningful plots of $\bar{U}=\bar{U}(\bar{a})$ with $M$ as a parameter, though this would certainly be interesting in principle. The basic problem is the tremendous range of 
$M$ ( 18 orders of magnitude) that is of interest. Our results do cover a range of $M$ from $O\left(10^{-9}\right.$ ) (for $R=200, W=2$ ) up to $O\left(10^{4}\right)$ (for $R=1, W=8$ ). However, only two (or, in rare cases, three) pairs of values of $\bar{a}, \bar{U}$ can be obtained from our results for any particular value of $M$ in this range, even with the help of interpolation. For example, our solutions for $R=50$ cover the range $O\left(10^{-6}\right)<M<O\left(10^{-4}\right)$, while $R=100$ covers $O\left(10^{-8}\right)<M<O\left(10^{-5}\right)$, and $R=200$ covers $O\left(10^{-9}\right)<M<O\left(10^{-6}\right)$, so that results for no more than two or three cases are available at any fixed $M$, even with interpolation. For lower values of $R$, only one case exists for any fixed $M$.

Thus the most that we can say about $\bar{U}$ as a function of $\bar{a}$ is some limited conclusions for fixed $M$ in the intermediate range $M=O\left(10^{-7}-10^{-5}\right)$. The most significant fact is the existence of regions in which the drag coefficient increases with increase of $R$ for given $M$, in qualitative agreement with experimental results for intermediate-size bubbles in low- $M$ liquids. An example is the case $R=100, W=5$, for which $C_{\mathrm{D}}=1.02$, and $R=200, W=10$, where $C_{\mathrm{D}}=1.64$. In both cases $M=O\left(10^{-6}\right)$.

It is noteworthy that the results reported here for the drag coefficient are in quite good accord with the boundary-layer solution of Moore (1965). For $R=200$ and $W=0$ and $W=2$ Moore obtains $C_{\mathrm{D}}=0.20$ and 0.37 respectively, while we obtain $C_{\mathrm{D}}=0.22$ and 0.33 . As we shall see in $\S 4.2$, there is no discernible separation for either of these cases, and this is in accord with Moore's (1965) analysis for $R \gg 1, W=O(1)$, in which the flow is assumed to remain attached. For either lower $R$ or higher $W$ (with $R=200$ ) the agreement with Moore's theory is much worse. For higher values of $W$ this is presumably a consequence of separation near the rear stagnation point, and large errors in Moore's approximate spheroidal shape (see \$4.2).

\subsection{Bubble shape}

The computed bubble shapes for all cases considered, except $R=0.5$, are shown in figure 2. Results for $R=0.5$ are given in figure 3, along with the shapes calculated for the same cases from the asymptotic formula of Taylor \& Acrivos (1964). The Taylor-Acrivos solution is derived under the asymptotic restrictions $R \ll 1, W \ll 1$ and $R^{2} \ll W$. According to Bhaga \& Weber (1981), experimentally observed bubble shapes agree with Taylor \& Acrivos (1964) for $R<0.5, W<1$, and the values of $W$ and $R$ in figure 3 were chosen purposely to be near this experimental limit. Examining figure 3, it is evident that the agreement between our solution and the Taylor-Acrivos theory is quite good at $R=0.5, W=0.5$, where the deformation is very small, but not so good at $R=0.5, W=1$. Although the Taylor-Acrivos solution was extended by Brignell (1973) to higher order in the deformation magnitude, Brignell's solution for $R=0.5, W \leqslant 1$ is extremely close (within $1 \%$ ) to the Taylor-Acrivos theory is quite good at $R=0.5, W=0.5$, where the deformation is figure 2 , beginning with the cases for $R \leqslant 20$ where $M$ is larger than $4 \times 10^{-3}$ for all but the smaller values of $W$ at $R=20$.

We may first note that, for $R \leqslant 20$, the progression of bubble shapes with increase of $W$ is in accord with qualitative 'expectations' based on the most commonly reported experimental observations, namely, spherical to oblate-ellipsoid to more complicated 'oblate' shapes with indentation and/or flattening at the rear. $\dagger$ Furthermore, as $W$ reaches $O(15)$, for $R \leqslant O(20)$, the shape of the bubble becomes visually

+ It should be kept in mind, however, that typical experiments do not hold either $R$ or $W$ constant, but rather the parameter $M$, with the independent dimensional parameter being the bubble volume. An increase in bubble volume with $M$ held constant yields an increase in $W$ proportional to $U^{2} a$ and an increase in $R$ proportional to $U a$. 


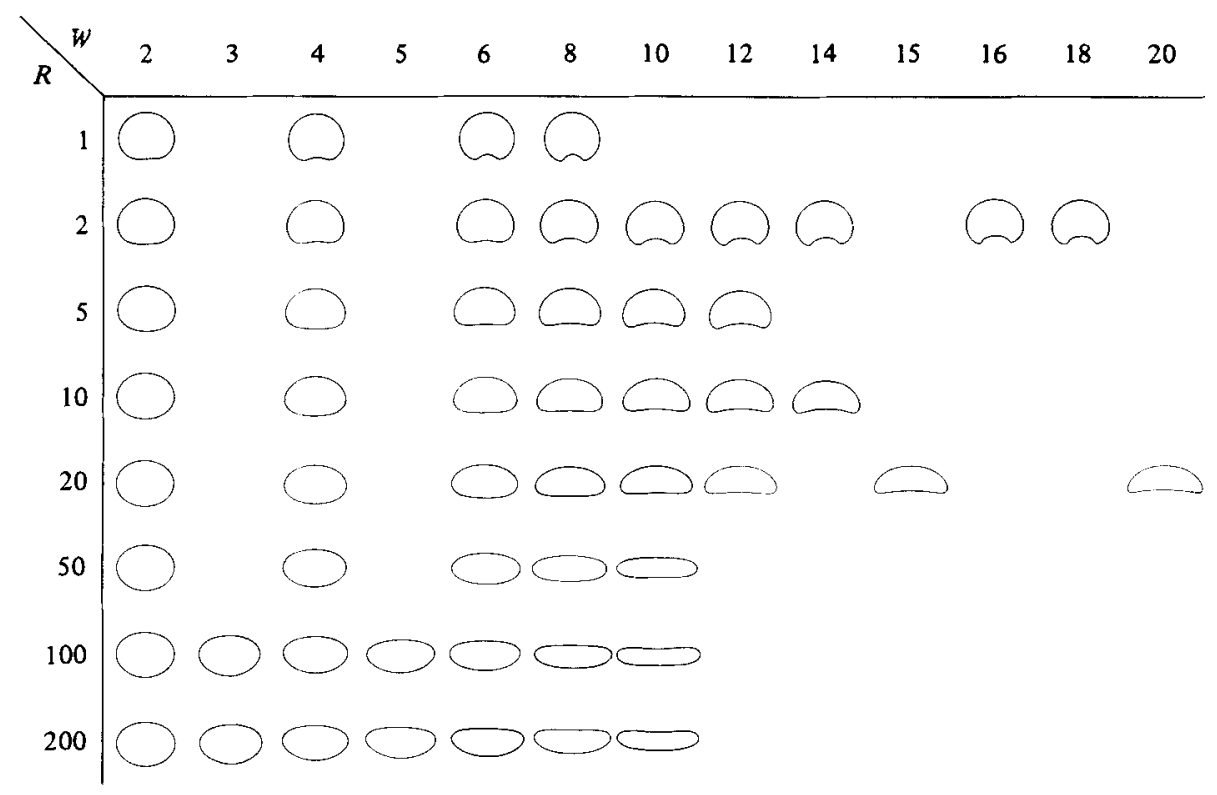

Figure 2. Computed steady, axisymmetric shapes of rising bubbles as a function of $R$ and $W$.

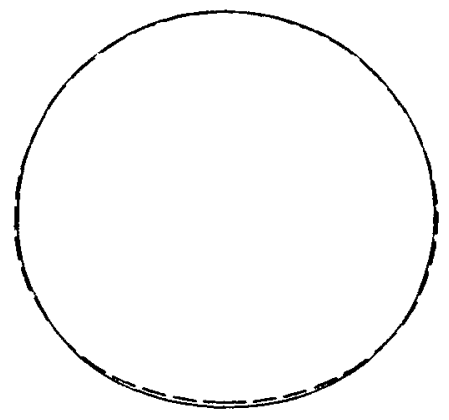

$R=0.5, W=0.5$

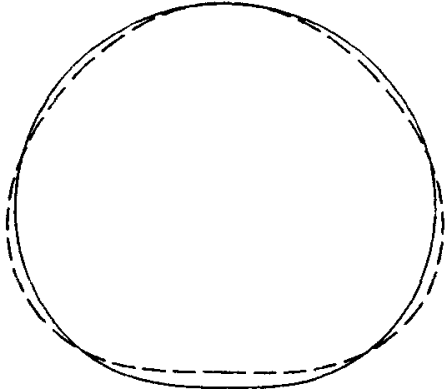

$R=0.5, W=1.0$

Figure 3. Comparison of the present results for bubble shape (solid line) with the asymptotic solution of Taylor \& Acrivos (1964) (broken line).

independent of $W$, in agreement with the experimental observations of Bhaga \& Weber (1981). It may be noted that direct comparison with the photographs of Bhaga and Weber is possible in some cases, e.g. our result for $R=2, W=16$ with their photo for $R=2.47$ and $W=16.5$ (their figure $3 a$ ), but the majority of their experimental results are for Weber numbers that are very high compared with those attained in the present work.

Although the bubble shapes for $W \geqslant O(15)$ appear visually independent of $W$ for any fixed $R \leqslant 20$, as noted above, our computations show that the influence of $W$ does still persist in this range in the vicinity of the bubble rim where the local curvature continues to increase with $W$. Evidently, two bubbles may have quite different local curvatures at the rim and still have almost-identical overall shapes. The local differences in curvature with increase of $W$ are, however, quite important for the flow fields, which continue to change with $W$ even after the overall bubble 
shape is visually constant - it may be seen from condition (8) of Part 1 that the amount of vorticity generated at any point on the bubble surface is proportional to the local curvature. The resultant changes in the vorticity and velocity fields with $W$ (see figure 5 for $R=20$ ) do not, however, seem to feed back to have a strong influence on the bubble shape.

In its present form, our numerical technique is not well suited for handling very high surface curvature. We therefore did not compute any solutions for $W>20$, and cannot do more than speculate as to the asymptotic nature of the flow for large $W$ and $R \leqslant 20$. In the low $R$ range it appears likely that the final asymptotic shape will not differ much, in an overall sense, from the highest- $W$ results of figure 2 . However, the curvature at the rim and consequently the amount of vorticity in the wake may well grow indefinitely with $W$. The existence of 'skirts' at the rim (cf. Hnat \& Buckmaster 1976) may suggest that a value of $W$ exists for any $R$ beyond which surface tension can no longer balance the local 'pulling' action of dynamic forces at the rim, but this is pure speculation at the present time.

In spite of the fact that shapes in which the deformation is strongest at the rear (eventually leading to spherical caps) have become accepted as the 'natural' mode of deformation, it is not at all obvious why these shapes should occur. At steady state any deformation is a consequence of local imbalances between hydrostatic and dynamic (normal) stresses at the bubble surface, which must, on the other hand, balance exactly in a certain global (i.e. integral) sense in order that the resultant force on the bubble be zero. Only for $R=0$ can we anticipate the bubble shape. In this case, the dynamic forces vary linearly with vertical position (i.e. with $x$ in our notation) for a spherical bubble, but not for any other shape that is nearly spherical (Kojima, Hinch \& Acrivos 1984). Thus, since the hydrostatic forces always vary linearly with $x$, it is evident that the bubble must be spherical in creeping flow for any value of $W$. For non-zero Reynolds number, on the other hand, the dynamic (normal) stresses do not vary linearly with $x$ when the shape is spherical, and the bubble deforms. However, it is extremely difficult to anticipate the details of the local imbalance between the static and dynamic normal stresses, and it is therefore equally difficult to develop any a priori prediction of the shape for particular values of $R$ and $W$. For example, examination of the surface distributions of hydrostatic pressure, dynamic pressure and the normal component of viscous stress, given in figure 4 for $R=2, W=16$, shows that the hydrostatic pressure contribution is locally dominant at the rear of the bubble, thus leading to the observed indentation in that part of the bubble (see figure 2). However, we see no a priori basis to anticipate the dominance of static-pressure contributions in this region. Another striking feature of the bubble shapes for high $W$ and $R \leqslant 20$ is that they can be closely approximated by intersecting segments of two spheres of different radii. This means that two strongly varying functions (the hydrostatic pressure and the dynamic normal stress) add up to one of two constant values everywhere on the bubble surface except in the local vicinity of the rim (see figure 4 for $R=2, W=16$ ). Again, it is not likely that this observed form of the difference between dynamic and static normal stress contributions could have been anticipated $a$ priori.

Let us now consider the results for bubble shape at the higher Reynolds numbers, $R \geqslant 50$. As can be seen from figure 2 , at these Reynolds numbers the (front) stagnation pressure becomes dominant and the bubble initially flattens more at the front than at the back. Eventually, for $R=100$ and 200 , the bubble actually develops an indentation in this area. Miksis et al. (1981) have also found bubble shapes in potential flow with an indentation at the front, but in that case the bubble shape must 


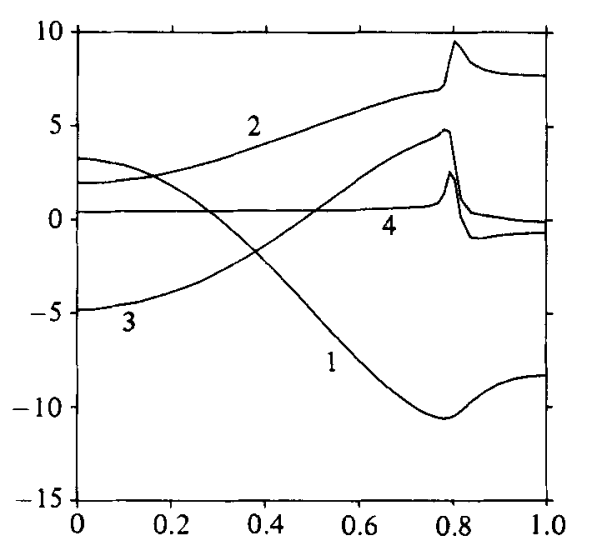

Relative distance from the front stagnation point

$$
R=2, W=16
$$

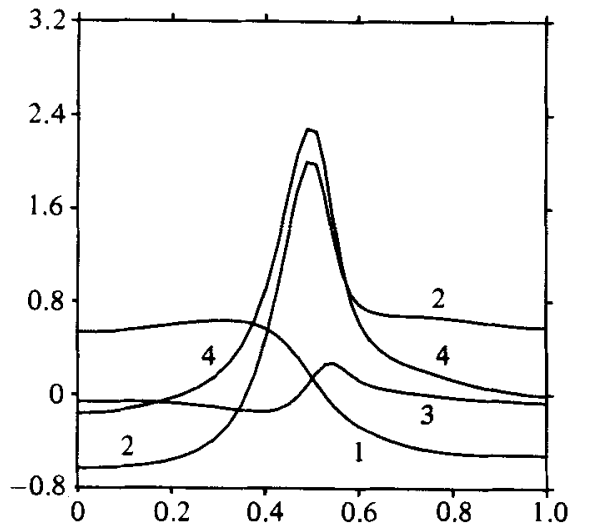

Relative distance from the front stagnation point

$$
R=100, W=10
$$

Fiaure 4. Surface distributions of pressure and viscous normal stress as functions of the (relative) distance from the front stagnation point. 1 , the hydrostatic pressure $-p_{\mathrm{hs}} ; 2$, the dynamic pressure $-p_{\text {dyn }} ; 3$, viscous normal stress $(8 / R) e_{\xi \xi} ; 4$, total normal stress $\tau_{\xi \xi}$, i.e. the sum of $(1)$, (2) and (3).

remain fore-aft symmetric so that the overall shape resembles a donut without the hole. In the real viscous flow, the rear stagnation pressure does not reach the same value as the front stagnation pressure (see figure 4 for $R=100, W=10$ ), and the deformation is greater in the front, as shown in figure 2. The case of $R=50$ lies approximately on the border between the two types of deformation: one characteristic of lower $R$, with stronger deformation in the rear, and another characteristic of higher $R$, with stronger deformation in the front. Consequently, at $R=50$ the bubbles almost preserve fore-aft symmetry, leading at higher Weber numbers to the shapes that Bhaga \& Weber (1981) termed 'disk-like' (see the photograph in their figure $2(c)$ for $R=55.3, W=15.4$ ). They note that these bubbles wobbled as they rose. Observations of saucer-like shapes such as those illustrated in figure 2 for $R \geqslant 100$ and $W=O(10)$ have also been reported in the literature (see e.g. Lane \& Green 1956), but we were unable to find any photographs for comparison. It is quite likely that this is a consequence of the fact that the real bubbles do not rise steadily at the highest Reynolds and Weber numbers that we attained in our computations (see $\$ 5$ below).

An extremely interesting feature of the present solutions is the evolution with increasing Weber number and fixed $R \geqslant 100$ of flatter and flatter shapes with an increasing indentation in front. It is possible, based on the trends of these solutions, that a limit point will be reached at some critical $W$ (dependent on $R$ ), beyond which no further solutions of this branch exist. Physically, such a limit point will occur if the dynamic forces caused by increased curvature increase faster than surface-tension forces, at least locally, so that an equilibrium shape is not possible. The suggestion that a limit point may occur at larger $W$ is supported by two pieces of evidence. First, precisely this type of result was found for potential flow past axisymmetric bubbles by Miksis et al. (1981), with steady shapes at subcritical values of $W<3.2$ which were qualitatively similar to those obtained here for $R=100$ and $R=200$ (though with fore-aft symmetry). Secondly, it is known from experimental studies that sphericalcap shapes occur for $W \geqslant O(30)$, and the branch of solutions found here does not seem likely to revert to a spherical-cap form for larger values of $W$. Unfortunately, restrictions in the present solution technique prevent us from extending the solutions 
at $R=100$ and 200 beyond $W=10$, and we cannot prove that solutions of the branch attained here do not exist for $W>W_{\mathrm{c}}$, nor can we provide a numerical estimate of the critical Weber number $W_{\mathrm{e}}$. Part of the problem is that the rate of convergence using our iterative algorithm for bubble shape slows considerably at these higher values of $R$ and $W$. In addition, the $40 \times 40$ grid is not sufficiently fine to merit extension of the present solutions to larger $R$ or $W$, while the use of a finer grid is currently too costly.

The fact that we cannot yet obtain results at larger $W$ does not, however, diminish the potential value of extending further the range of steady axisymmetric solutions. The use of such solutions in the present problem to establish the non-existence of the branch of flattened shapes beyond some critical $W=W_{\mathrm{c}}(R)$ would presumably signal the onset of 'shape instabilities', which may account for the violent fluctuations and irregularities of shape that are observed experimentally before the bubble attains a spherical cap shape (Saffman 1956). Of course the existence of steady solutions for $W<W_{\mathrm{c}}(R)$ is no guarantee of their stability. This is a subject of current research. Experimental evidence from earlier studies would seem to provide strong evidence of instability prior to $W=W_{\mathrm{c}}(R)$, and to suggest strongly that 'shape instability' associated with the non-existence of steady axisymmetric solutions for bubble shape should be distinguished from non-axisymmetric shapes caused by non-rectilinear trajectories that result from intrinsically unsteady wake flows (termed "path instability'). Experimental studies show that 'path instability' occurs at relatively low $W \sim 3$ when the bubble is still only slightly deformed (cf. Hartunian \& Sears 1957), and is of a completely different nature than the 'instability of shape' associated with a large, supercritical deformation. We will discuss this point in more detail in $\$ 5$. However, in our opinion, the fact that Miksis et al. (1981) found 'shape instability' in potential flow at Weber numbers very near the critical value observed in experiments for 'path instability' is purely coincidental. 'Path instability' cannot be predicted with the numerical procedure used here if the flow is assumed axisymmetric and steady from the outset (the same is true of the potential flow solutions of Miksis et al. 1981). 'Shape instability', on the other hand, implies non-existence of steady axisymmetric bubble shapes above some Weber number, and this will manifest itself quite clearly under the above assumptions.

It is relevant to note that the branch of solutions exhibiting increased flattening with $W$ was generated here by holding $R$ constant and gradually increasing $W$ starting with a sphere for $W=0$. It is quite possible that the spherical-cap branch of solutions for $R \geqslant O(100)$ could be reached numerically by first computing spherical-cap bubbles for $R=O(20)$ and $W=O(30)$ - where they must presumably exist as an extension of our present solution for $R=20, W=20$ - and then increasing $R$ while holding $W$ constant. However, we have not yet attempted to attain spherical caps by this route, pending changes in the numerical scheme to better accommodate local regions of high surface curvature.

Finally, it may be noticed from figure 2 that the dependence of the shape on Reynolds number becomes rather weak for $R \geqslant O(100)$. It is not clear, however, if this means that the solution is near its asymptotic form for $R \rightarrow \infty$ or that the values of $R=O(100)$ correspond to a 'plateau' region in the dependence of the shape and flow structure on the Reynolds number. Nor can one discard the possibility that this behaviour is (at least partly) an artifact of the numerical scheme working at its limits of accuracy. 


\subsection{Flow structure-separation at a free surface}

In different branches of fluid mechanics flows with free surfaces at high Reynolds numbers are treated as irrotational. This is a good approximation if the flow does not separate, since then the velocity at the free surface will differ from its irrotational value by a small amount proportional to $R^{-\frac{1}{2}}$ (see Batchelor 1967, $\$ 5.14$ ). If the flow separates, however, the entire structure of the flow field will be changed and the assumption of irrotational flow is not useful. It is therefore quite important to understand if such separation may indeed occur at a free surface, and under what conditions. We are not aware of any theoretical or numerical work in this area. There appears to exist a belief that a flow does not separate from a smooth free surface but may separate if a free surface has a sharp edge (see e.g. Batchelor 1967, $\$ 5.14$ and 6.11).

At intermediate Reynolds numbers, flow separation can be studied numerically, and the present problem provides an excellent opportunity for such a study. Our results (see figure 5) show that separation not only occurs at a smooth free surface, but that the size of the separated-flow region may be very substantial. The onset of separation depends on both Reynolds and Weber numbers. Very roughly, it occurs at $W \approx 9$ for $R=10$, at $W \approx 7$ for $R=20$, at $W \approx 5$ for $R=50$, at $W \approx 4$ for $R=100$, and at $W$ between 3 and 4 for $R=200$. The size of the separated region grows with both $R$ and $W$, though for $R \geqslant O(100)$ the dependence of this size on $R$ is rather weak, similar to the situation for the shape of the bubble, discussed at the end of $\$ 4.2$. The present solutions also show that the dividing zero-streamline leaves the surface of the bubble at a large angle, though the fine structure of solution near the point of detachment cannot be studied on our relatively coarse grid.

An obvious question is whether the streamline patterns and shapes shown in figure 5 are realistic, especially since the existence of separation from a smooth zeroshear-stress surface is not, perhaps, easily accepted by all workers in the field. To answer this question, the most effective course is comparison of our calculated solutions with actual photographs. We could only find one example of a photograph that clearly showed a standing eddy structure downstream and also had values of $R$ and $W$ matching closely one of our numerical cases. A comparison of the computed and experimental bubble shape and wake structure is shown in figure 6 . As noted, the photograph was taken from the study of Hnat \& Buckmaster (1976). The experimental and numerical results for both shape and streamline pattern compare extremely well. It should be noted that the indentation at the rear of the bubble is visible in the original published photo, though somewhat obscured by the fact that we look through the edge of the bubble. It is, however, more difficult to discern in our reproduction of the Hnat-Buckmaster photo.

How can one explain the appearance of separation in this flow with a free surface? The most frequently cited explanation for the existence of a standing eddy in flow past a solid body is that it is a consequence of boundary-layer separation, the latter occurring because the no-slip condition at the body surface causes a deficit of momentum in the fluid nearest the boundary relative to what it would have in potential flow. Thus, the fluid in this boundary region does not retain enough kinetic energy to overcome the pressure rise at the rear of the body, and breaks away into the main body of fluid. Evidently, in separation at a zero-shear-stress interface this type of argument cannot be used. We believe that the best explanation for the appearance of standing eddies, and/or boundary-layer separation, is the evolutiontype argument due to Batchelor $(1967, \S 4.12)$, according to which the boundary-layer separation is a consequence of the development of standing eddies behind the body 


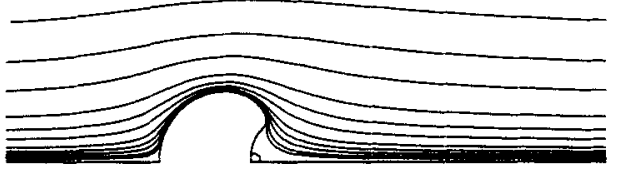

$R=2, W=12$

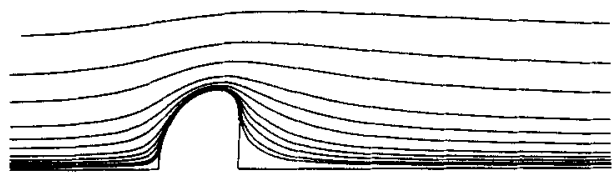

$R=10, W=8$

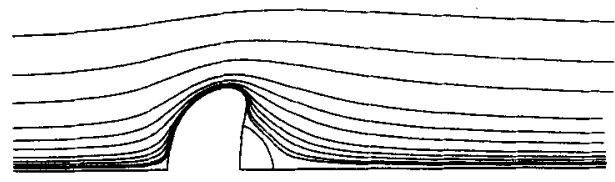

$R=10, W=12$

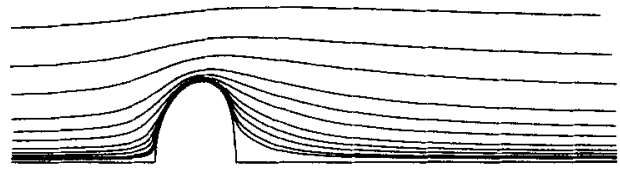

$R=20, W=6$

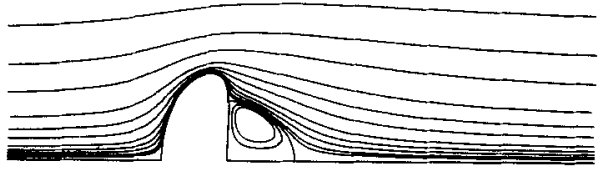

$R=20, W=10$

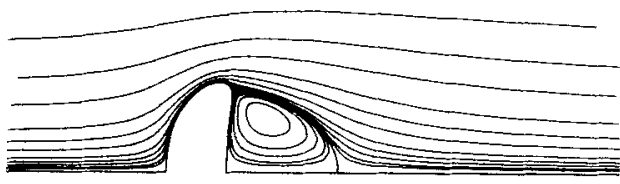

$R=20, W=15$

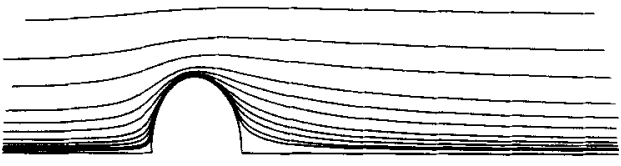

$R=50, W=4$

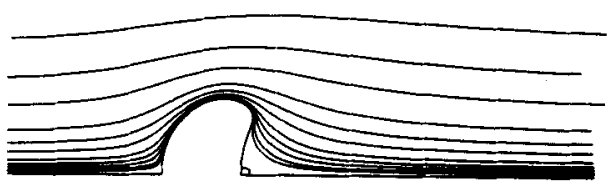

$R=5, W=12$

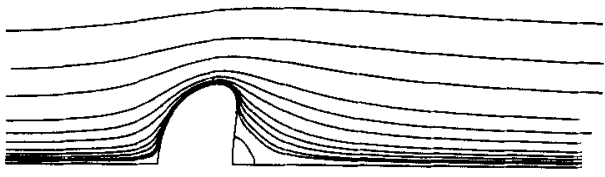

$R=10, W=10$

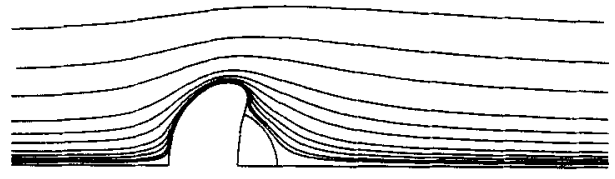

$R=10, W=14$

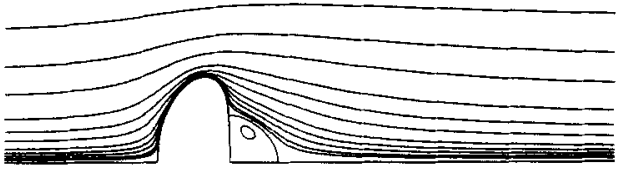

$R=20, W=8$

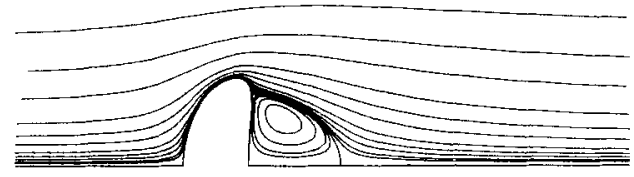

$R=20, W=12$

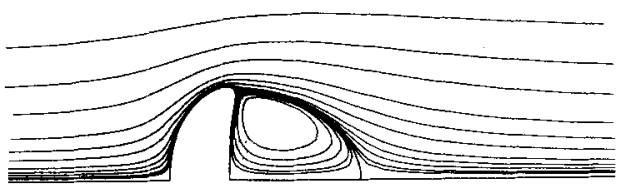

$R=20, W=20$

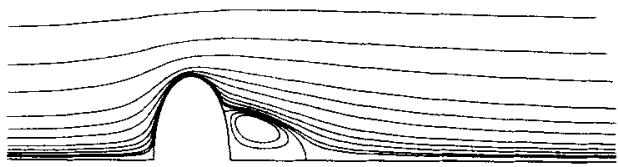

$R=50, W=6$

Figure 5. For caption see facing page. 


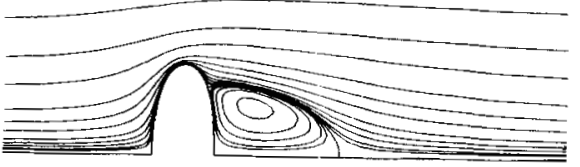

$R=50, W=8$

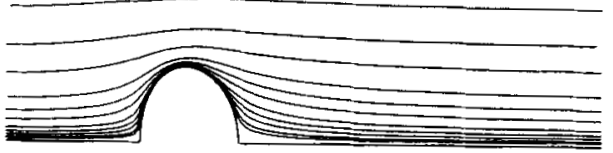

$R=100, W=3$

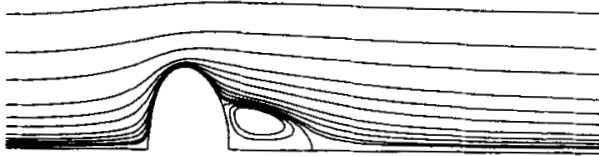

$R=100, W=5$

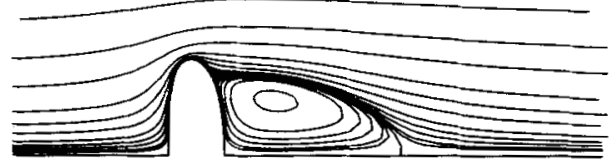

$R=100, W=8$

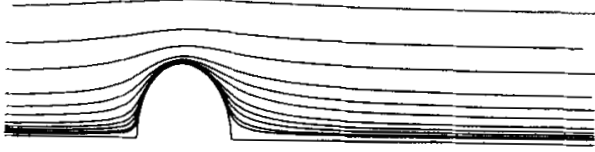

$R=200, W=3$

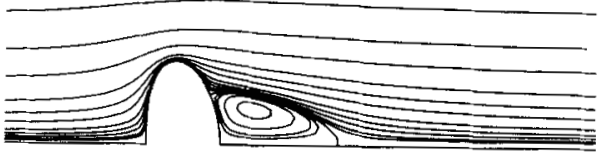

$$
R=200, W=5
$$

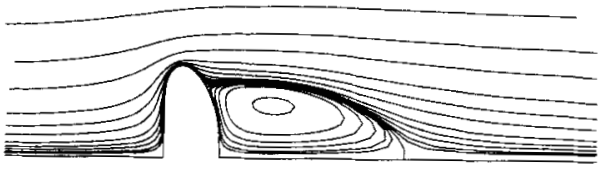

$$
R=200, W=8
$$

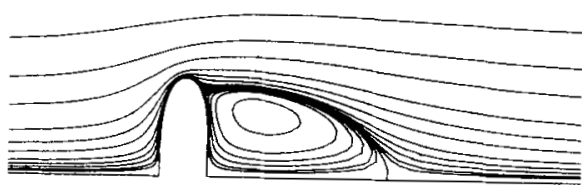

$R=50, W=10$

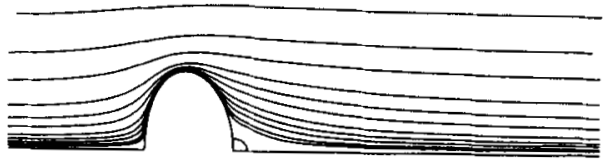

$R=100, W=4$

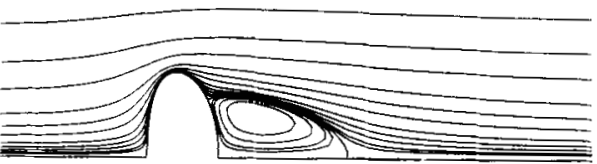

$R=100, W=6$

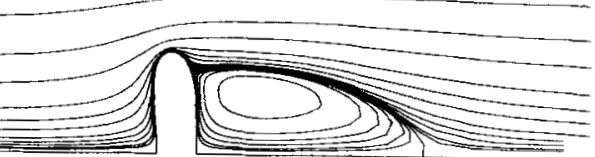

$R=100, W=10$

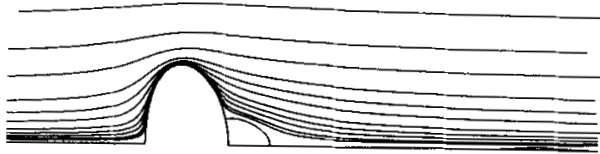

$R=200, W=4$

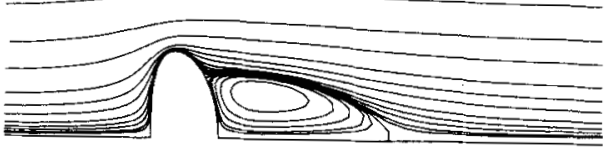

$R=200, W=6$

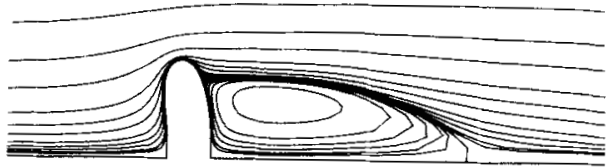

$R=200, W=10$

Figure 5. Flow separation at a free surface: the standing-eddy structure behind the bubble. The contour values for the stream function are $0, \pm 0.001, \pm 0.002, \pm 0.005, \pm 0.01, \pm 0.02$, etc. 


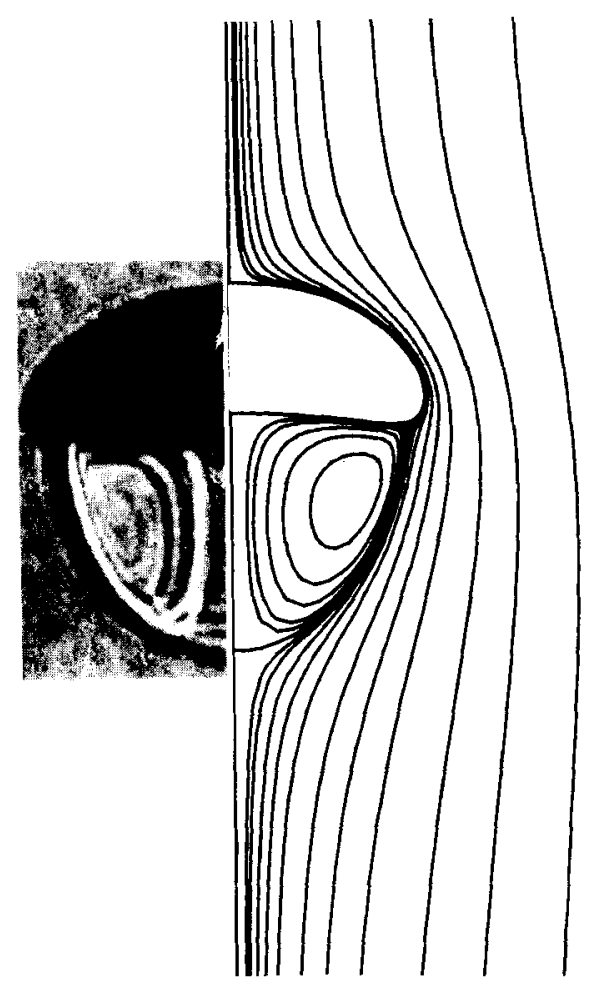

Froure 6. Comparison of the experimental photograph by Hnat \& Buckmaster (1976) for $R=19.4, W=15.3\left(C_{\mathrm{D}}=3.44\right)$ with present results for $R=20, W=15\left(C_{\mathrm{D}}=3.55\right)$.

due to the accumulation of vorticity brought to this area by convection, rather than vice versa. A crucial piece of evidence in support of this view is the fact that while separation in laminar flow is always accompanied by standing eddies, the standing eddies behind a body may exist without separation, as observed experimentally for a bluff body with base bleed by Leal \& Acrivos (1969), and found numerically for a spherical viscous drop by Rivkind \& Ryskin (1976). Clearly, the eddies are primary and the separation secondary. However, unless something prevents the standing eddy from attachment (base bleed, internal motion in the drop), the eddy will attach and thus cause separation.

According to the viewpoint expressed above, the key condition for existence of standing eddies (and thence separation) is the generation of vorticity at a sufficient rate at the bubble surface. As is evident from condition (8) of Part 1, this implies the existence of sufficiently large curvature, i.e. bubble deformation. Since the shape of a bubble depends on both the Reynolds and Weber numbers it is difficult to separate the effects of these parameters on the existence of standing eddies (and separation), especially in view of the fact that the Reynolds number is also indicative of the relative efficiencies of convection and diffusion in the transport of vorticity. It can be seen, however, that no separation occurs at any of the Reynolds numbers considered here when the bubble is spherical in shape (i.e. $W=0$ ) - a result also confirmed by earlier numerical studies due to Brabston \& Keller (1975) and Rivkind \& Ryskin (1976) for spherical bubbles. This is presumably due to the fact that the surface curvature and hence boundary vorticity is not sufficiently large for a bubble of spherical shape. As $W$ increases, however, bubble deformation increases, as does the maximum surface curvature and vorticity, and it is not surprising to find that 
separation occurs for a particular Reynolds number at some critical value of $W$ (dependent on $R$ ). Our numerical results show that the deformation need not be all that great to yield sufficient vorticity to give standing eddies; the maximum boundary vorticity at the onset of separation is of the order 4-10, being smaller for lower Reynolds numbers (i.e. for more bluff shapes). $\dagger$

Although experimental photographs have been published by a number of authors, including the Hnat-Buckmaster (1976) study cited earlier, which show clear evidence of separation, there has been a reluctance to accept this result as unequivocal evidence for separation from a free interface. In particular, in the experiments, it is never clear that the interface is completely free of surfactants, temperature gradients or other impurities which could change the boundary conditions and thus conceivably account for the existence of separation. The present numerical results, on the other hand, do satisfy the zero-shear-stress condition to within a small numerical error, and thus provide a clear demonstration of flow separation from a smooth free surface, at least for intermediate Reynolds numbers. Consequently, the usual assumption that flow near a smooth free surface will correspond closely to potential flow at high Reynolds numbers cannot be taken for granted, but requires additional investigation.

\section{Discussion}

Finally, in this section we consider some further implications of our numerical results for the conditions for existence of the unsteady and/or non-axisymmetric motions observed by Saffman (1956), Hartunian \& Sears (1957) and many other investigators. As noted in the description of experimental results, these motions consist, first, of zigzag or helical trajectories for sufficiently large Reynolds numbers and relatively small Weber numbers where the shape deformation is only moderate, and, secondly, violent chaotic fluctuations of shape with a nearly rectilinear path for more highly deformed, saucer-like bubbles at higher Weber number. The latter phenomenon has been tentatively associated with the non-existence of steady axisymmetric flattened shapes beyond a certain critical Weber number (see $\$ 4.2$ ). It was, in fact, suggested many years ago by Haberman \& Morton (1953) that the origin of the zigzag or helical path at lower Weber numbers was a consequence of vortex shedding from the bubble. However, this explanation was not readily accepted because it presumed the existence of a standing-vortex structure, and because lateral motions of similar magnitude had not been observed for rigid spheres even though rigid spheres and spherical bubbles should exhibit similar hydrodynamic resistance to lateral translational accelerations due to the added-mass effect (Saffman 1956). Later experiments with extremely light solid spheres by Preukschat (1962) and MacCready \& Jex (1964) did, however, show large-scale oscillations of path similar in character to those observed for bubbles, thus lending support to Haberman \& Morton's (1953) hypothesis. Nevertheless, the fundamental question remains as to why heavy and light bodies in a given fluid should exhibit differences in the amplitude of path oscillations when the effective mass (i.e. actual + added mass) differs by only an $O(1)$ numerical factor. We believe that the reason lies in the large differences in moment of inertia, which, for spheres, is directly proportional to the particle density (the added moment of inertia for a sphere is zero). The lateral force associated with vortex shedding is a consequence of the periodic Magnus force arising from interac-

$\dagger$ This is the same order of magnitude for the surface vorticity at the point of onset of standing eddies (i.e. separation) that one obtains for a solid sphere, though in that case the surface vorticity increases as the Reynolds number increases, leading to a minimum-Reynolds-number criterion for the existence of separated flow. 
tion of the main flow past the sphere and the circulation around it (the latter being induced each time an amount of vorticity is shed and being of opposite sign to this vorticity). The moment of inertia is important because the induced circulation around the sphere will be strongly damped by viscous effects unless the sphere participates readily in the rotation. The latter will be true of a bubble or a 'hollow' sphere, but not for a solid sphere, since the moment of inertia for a sphere is proportional to its density.

Now, for vortex shedding to occur, two obvious conditions should be met: the standing eddy must exist behind the body, and the Reynolds number must exceed the critical value $R_{\mathrm{c}}$ at which the motion in the wake becomes unstable. In the case of a rigid bluff body the standing-eddy structure always appears at Reynolds numbers considerably below $R_{\mathrm{c}}$, which is $O(200)$ (see e.g. Nakamura 1976, for spheres: Willmarth, Hawk \& Harvey 1964, for disks). For bubbles, on the other hand, our solutions show that separation is not found for any Reynolds number $(\leqslant 200)$ when the shape is spherical or near-spherical. Thus, in spite of the fact that a Reynolds number may be reached at which vortex shedding would occur if a vortex structure existed, our solutions suggest that there will be little or no departure from a rectilinear path until a sufficiently large $W$ is reached to obtain the necessary downstream eddy structure.

These implications of the present solutions appear to provide a basis for understanding the experimental results of Hartunian \& Sears (1957), who found two distinct criteria for the change from a rectilinear to zigzag or helical path:

(a) a critical Reynolds number $R \approx 200$ for impure liquids;

(b) a critical Weber number $W \approx 3.2$ for pure liquids.

Apparently, in the impure liquids, the conditions on the surface of the bubble are sufficiently close to no-slip that the vortex ring forms even for bubble shapes close to spherical when the Reynolds number is below $R_{\mathrm{c}}$, and the usual criterion for onset of vortex shedding from a solid is obtained. In pure liquids, on the other hand, the free-surface conditions are relevant, and the condition for onset of zigzag for any sufficiently large Reynolds number is the existence of a sufficiently large Weber number to allow formation of the downstream eddy structure. Our numerical solutions show that the Weber number required for existence of separation is somewhat dependent upon the Reynolds number, but decreases to a value of $O(3-4)$ for $R=200$. This is close to the value reported for onset of zigzag in clean fluids by Hartunian \& Sears (1957). At lower Reynolds numbers it is unlikely that the wake structure becomes unstable (based on the necessary critical $R$ for spheres and disks cited earlier), though instability of shape may be expected for $R=O(50-100)$, where the flattened branch of steady solutions suggests a critical Weber number for non-existence of steady axisymmetric shapes. We may also note that the existence of a perfectly pure interface is difficult to achieve (especially for water) in an experiment, and any small departure to a slightly contaminated surface will tend to lead to separation at lower $W$ than predicted here for the perfectly clean, zeroshear-stress interface. This may account for the fact that the Hartunian \& Sears (1957) critical Weber number was only 3.2, while the present numerical results at $R=200$ would suggest the need for $W=O(5-6)$ in order to have not only onset of separation but some development of the standing-vortex structure behind the bubble. It may be noted, in support of this contention, that Tsuge \& Hibino (1977) report higher critical Weber numbers for onset of zigzag motions, up to $O(5)$, for bubbles in highly purified organic liquids. 
This work was supported by a grant from the Fluid Mechanics Program of the National Science Foundation.

\section{REFERENCES}

Batchelor, G. K. 1967 An Introduction to Fluid Dynamics. Cambridge University Press.

BhaGA, D. \& WeBER, M. E. 1981 Bubbles in viscous liquids: shapes, wakes and velocities. J. Fluid Mech. 105, 61-85.

Brabston, D. C. \& Keller, H. B. 1975 Viscous flows past spherical gas bubbles. J. Fluid Mech. 69, $179-189$.

BrignelL, A. S. 1973 The deformation of a liquid drop at small Reynolds number. $Q . J$. Mech. Appl. Maths 26, 99-107.

Collins, R. 1966 A second approximation for the velocity of a large gas bubble rising in an infinite fluid. J. Fluid Mech. 25, 469-480.

Davies, R. M. \& TAYlor, G. I. 1950 The mechanics of large bubbles rising through extended liquids and through liquids in tubes. Proc. $R$. Soc. Lond. A 200, 375-390.

Haberman, W. L. \& Morton, R. K. 1953 An experimental investigation of the drag and shape of air bubbles rising in various liquids. David Taylor Model Basin Rep. 802.

Hartunian, R. A. \& Sears, W. R. 1957 On the instability of small gas bubbles moving uniformly in various liquids. J. Fluid Mech. 3, 27-47.

Hrat, J. G. \& Buckmaster, J. D. 1976 Spherical cap bubbles and skirt formation. Phys. Fluids 19. $182-194$.

Kojtma, M., Hinch, E. J. \& Acrivos, A. 1984 The formation and expansion of a toroidal drop moving in a viscous fluid. Phys. Fluids 27, 19-32.

LANE, W. R. \& GREen, H. L. 1956 The mechanics of drops and bubbles. In Surveys in Mechanics (ed. G. K. Batchelor \& R. M. Davies), pp. 162-215. Cambridge University Press.

Leat, L. G. \& Acrivos, A. 1969 The effect of base bleed on the steady separated flow past bluff objects. J. Fluid Mech. 39, 735-752.

MacCready, P. B. \& Jex, H. R. 1964 Study of sphere motion and balloon wind sensors. NASA $T M \times-53089$.

Mrksis, M., Vanden-Broeck, J.-M. \& Keller, J. B. 1981 Axisymmetric bubble or drop in a uniform flow. J. Fluid Mech. 108, 89-100.

Miksis, M., Vanden-Broeck, J.-M. \& Keller, J. B. 1982 Rising bubbles. J. Fluid Mech. 123, 31-41.

Moore, D. W. 1959 The rise of a gas bubble in a viscous liquid. J. Fluid Mech. 6, 113-130.

Moore, D. W. 1965 The velocity of rise of distorted gas bubbles in a liquid of small viscosity. J. Fluid Mech. 23, 749-766.

Nakamura, I. 1976 Steady wake behind a sphere. Phys. Fluids 19, 5-8

Preukschat, A. W. 1962 Measurements of drag coefficients for falling and rising spheres in free motion. M.S. thesis, Department of Aeronautical Engineering, California Institute of Technology.

Rivkind, V. Y. \& Ryskin, G. 1976 Flow structure in motion of a spherical drop in a fluid medium at intermediate Reynolds numbers. Fluid Dyn. 11, 5-12.

Ryskin, G. \& Leat, L. G. 1984 Numerical solution of free-boundary problems in fluid mechanics. Part 1. The finite-difference technique. J. Fluid Mech. 148, 1-17.

SAFFMAN, P. G. 1956 On the rise of small air bubbles in water. J. Fluid Mech. 1, 249-275.

TAYlor, T. D. \& Acrivos, A. 1964 On the deformation and drag of a falling viscous drop at low Reynolds number. J. Fluid Mech. 18, 466-476.

Tsuge, H. \& Hibino, S. I. 1977 Onset conditions of oscillatory motion of single gas-bubbles rising in various liquids. J. Chem. Engng Japan 10, 66-68.

Wegener, P. P. \& Parlange, J.-Y. 1973 Spherical-cap bubbles. Ann. Rev. Fluid Mech. 5, 79-100.

Willmarth, W. W., Hawk, N. E.\& Harvey, R. L. 1964 Steady and unsteady motions and wakes of freely falling disks. Phys. Fluids 7, 197-208. 